\title{
Bridge the gap between high school systems with less than twelve years of schooling and European Universities
}

\author{
Francesco Floris ${ }^{1}$, Marina Marchisio ${ }^{1}$, Carla Marello ${ }^{2}$, Lorenza Operti ${ }^{3}$
}

${ }^{1}$ Department of Mathematics, Università degli Studi di Torino, Italia, ${ }^{2}$ Department of Languages, Università degli Studi di Torino, Italia, ${ }^{3}$ Dipartimento of Chemistry, Università degli Studi di Torino, Italia.

\begin{abstract}
The phenomenon of globalization that concerns the modern era, pushed by technological evolution, has led to several changes in the field of education. Not only are education policies of the single States adapting by directing towards European models: the possibility for a student to choose a university is increasing all over the world, too. In order to facilitate students who want to enroll at a European university and who come from countries with less than 12 years of compulsory schooling, the University of Turin designed the Foundation Programme. This is an additional year that allows to earn 60 or 30 ECTS to reach the 12 years of schooling, a basic requirement for university access in Europe. The main feature of this project is that it is delivered online; this allows to reduce the costs of enrollment and allows students to attend it directly from home in their country. The design, structure and methodologies of the project are described and discussed in this article.
\end{abstract}

Keywords: E-learning; Foundation Programme; Foundation Year; Globalization in education. 


\section{Introduction}

To talk about globalization means to talk about everything concerning the planet, a phenomenon that is not new in human history, but which, especially nowadays, where technological progress dominates, has taken on a much wider dimension than in the past. Globalization has led to "in political terms, a certain loss of nation-state sovereignty, or at least the erosion of national autonomy, and, correspondingly, a weakening of the notion of the "citizen" as a unified and unifying concept, a concept that can be characterized by precise roles, rights, obligations, and status" (Burbules \& Torres, 2000). Due to the strong interaction between state and education, globalization has also affected the educational field (Isaxanli, 2018), and an increasing number of countries are adopting a school system similar to the European or American model. Globalization has also led to an increase in internationalization in terms of mobility of students, teachers, Erasmus exchanges and internships. In Italy, data of the National Student Registry (ANS) show that the number of foreign students enrolling at an Italian university has been constantly increasing, and in the academic year 2017/18 it was over 23,000 , which is about $6 \%$ of the total number of the students enrolled at an Italian university, with an increase of $8 \%$ when compared to a.y. 2016/17. The number of foreign students enrolled at the University of Turin is 6\%, in line with the national average. Some of these students come from countries where the 12 years of schooling are not required as for the enrollment at an Italian university. To facilitate the integration of their pre-university education, the University of Turin has created the Foundation Program, an additional year that allows the achievement of 30 or 60 ECTS, according to their needs. It is delivered completely online: this way, international students can attend the program from their homes, without the need of a stay permit and without the costs of the travel and stay in our country. Only the final exam that allows to certify the ECTS is to be taken at the university, right before enrollment.

\section{State of the art}

\subsection{Regulations for enrolling foreign students at an Italian university}

As indicated by the Ministry of Education, University and Research, the enrollment of a foreign student at an Italian university requires the delivery to consular representation and institutions of advanced education, of, among other documents, "The final title in original (or certified copy) of secondary studies obtained with at least 12 years of schooling, or substitutive certificate to all legal effects; the final title can be accompanied as an alternative and at the discretion of the single institution of higher education by certification issued by ENIC-NARIC centers, by certificates of foreign official bodies or by Declaration of Value" (MIUR, 2018). On the same document there is a reference to article 6 paragraph 1 of Ministerial Decree 270/2004 for what concerns the university area, and article 7 paragraph 2 
of Presidential Decree 212/2005 for the AFAM (Artistic, Musical and Choreutic Higher Education) area, which regulate the possibility for institutions of higher education to establish "preparatory education activities for admission to degree programs, offering preparatory courses (foundation course) even for a duration of less than one year, in order to meet the admission requirements for attending Italian higher education courses".

Several Italian universities have taken steps in this direction by activating this supplementary year, such as the University of Pavia, the University of Pisa, the University of Siena, and the University of Rome "Tor Vergata". What brings together these universities is the proposal of a foundation year provided either entirely face to face or through mixed activities both face to face and online. At a European level, the panorama appears to be much more diverse, both from the point of view of the objectives and from the point of view of the delivery of the Foundation Year, but there are numerous initiatives that allow foreign students to learn in a new study modality. The University of Turin has decided to set up its own introductory course in interactive mode completely online, self-paced, with the sole exception of the final exam, which has to be taken face to face.

\subsection{Digital education at the University of Turin}

The choice of entirely delivering the Foundation Programme via online courses was made for two main reasons. First of all, we wanted to try to cut the costs for the course attendance to a minimum by offering the possibility to attend it to people from disadvantaged regions, or to students who are not so much interested in enrolling at university as rather wishing to expand their cultural preparation or to learn more about Italian culture and language. The second reason is that an online course of this kind is part of the strategic action taken by the University of Turin to invest in digital education, a field in which it has gained experience and carried out research. In line with this, several initiatives were seized over the last years, with different goals and objectives. A first example is represented by Orient@mente, which provides all secondary school students with support in the preparation for the university and in a more informed choice of the academic path, by an open and free online platform (Barana, Bogino, Fioravera, Floris, Marchisio, 2018a; Barana et al., 2017a; Barana, Bogino, Fioravera, Marchisio, Rabellino, 2016a). The project Start@Unito pursues orientation purposes and offers a link between university and secondary school. It is an online platform containing numerous free access university courses that can be attended autonomously from the last years of secondary school and allow students to immediately acquire the university credits, upon passing the final face to face exam after enrollment. (Bruschi et al., 2018; Marchisio, Operti, Rabellino, Sacchet, 2018). While the previous initiatives target the students, there are also numerous online training activities for secondary school teachers. From a European perspective, in the SMART project - Science and Mathematics Advanced Research for a Good Teaching - two open online courses have been designed and implemented as intellectual outputs containing resources and useful activities for a continuous updating in 
the teaching of teachers of scientific disciplines. (Brancaccio, Esposito, Marchisio, Pardini, 2016). At a national level, the University of Turin is a scientific partner of the ministerial project "Problem Posing \& Solving", which promotes the creation of a community of learning and practice for teachers of the STEM disciplines of Italian secondary schools (Barana et al., 2017b; Brancaccio et al., 2015; Barana, Fioravera, Marchisio, Rabellino, 2017e).

\section{The project "Foundation Programme"}

The Foundation Programme is available at foundationprogramme.unito.it starting March 2019. It is in Italian and written in Italian, since half of the courses are on Italian language and culture. It offers two modules, one consisting of 30 ECTS and the other one consisting of 60 ECTS. The first 10 ECTS are completely open, so as to allow students to verify the actual interest for the path chosen whereas, in order to access the other courses, students need to enroll and pay the anticipated fee.

The design of the Foundation Program has been divided into different highly correlated phases: analysis of the student needs, definition of the educational objectives, the choice of the learning environment to be used and the summative evaluation of the path. A starting point for the choice of the design methodology was identified in the ELED (E-Learning Engagement Design) model (Czerkawski \& Lyman, 2016), an Instructional Design Framework for Student Engagement which provides strategies to maximize the participation of students in an online course.

a) Analysis of student's needs. A student enrolling in the Foudation Programme might encounter two main difficulties: language, and the possibility to attend classes in a face to face modality. To provide for the first necessity, all the courses are in Italian, with an intermediate level of the language (B1-B2) and 30 of the ECTS to be achieved belong to the "Italian language and culture" module. The B1-B2 level is not required for the enrollment in the Foundation Programme, but it is the recommended level of language in order to easily attend the supplementary year and it represents a minimum basis so as to attend lessons of any degree course in an Italian university. As for the difficulty of attending the course in face to face mode, the solution adopted was, as already mentioned, that of providing the course in online mode. On one hand, it allows students to attend the course from their countries in total autonomy and at their own pace, while also containing costs, since they only need to pay for enrollment. On the other hand, this modality required us to adopt various methodologies that are able to stimulate the student in learning, to help him/her overcome obstacles, to trigger cognitive and metacognitive processes, to have self-evaluation mechanisms that allow him/her to check his/her progress. Moreover, since the course is completely online, there is no minimum number of 
students required to be enrolled in order for the activation of the supplementary year, unlike some face to face courses.

b) Definition of educational objectives. The definition of educational objectives was made at different levels. The main educational objective of the course is to provide the student with the basic skills necessary for the enrollment in a university course, while each course was associated to two sub-levels of educational objectives. Of these two objectives, the first one regards the educational objectives of the single course, while the second one describes the specific objectives for each of the ECTS. This way, the student can consciously check his learning process, have clear all the steps and have more confidence in his/her progresses. This is of fundamental importance in the case of such online courses, where there is no tutoring or monitoring of the activity by a teacher. The choice of educational objectives was certainly a challenge for university teachers involved in carrying out the lessons, since they had to be designed for younger students with respect to their normal didactic activities, and therefore they had to reshape them both in terms of content and language.

c) Choice of the learning environment. Given the proven experience in the field of elearning gained by the University of Turin with the Moodle learning management system, the latter was chosen as a learning environment. According to Dixson (Dixson, 2010) there are no significant differences between the level of student involvement and the type of activity proposed (with a distinction between active activities, such as discussion forums or problem solving activities, and passive activities, such as reading educational material, video lessons, etc. ..), but what really maximizes the involvement of the students are the interactivity and the interactions (student-teacher, student-tutor and also student-student). Since the Foundation Programme is delivered in a self-paced modality, interactivity is one of the aspects that were carefully taken into account in its design. Tools that allow immediate and interactive feedback were adopted, together with adaptive courses based on ongoing assessments and problem solving activities for the contextualization of the notions presented. The Moodle platform is integrated with an Advanced Computing Environment (ACE) and an Automatic Assessment System (ASS) that allow to guide students through personalized paths (Barana, Conte, Fioravera, Marchisio, Rabellino, 2018b; Barana, Marchisio, 2016b; Barana, Marchisio, Rabellino, 2015).

d) Path evaluation. The summative evaluation at the end of the path is divided into two parts. The first part is the autonomous one that only concerns the student, to whom a certificate of attendance is given online, after passing the test with automatic evaluation at the end of the course. The second part is the face to face exam for the 
certification of the ECTS, to be passed in Turin, but recognized by any Italian university.

\subsection{Structure of the "Foundation Programme"}

Analyzing the needs of the students and taking into account the different number of ECTS needed for enrollment based on the years of schooling acquired, it was decided to provide the Foundation Program with two paths, one made of 30 ECTS and one made of 60 ECTS. The first one contains three compulsory modules: "Lingua e cultura italiana" (20 ECTS), "Letteratura Italiana” (5 ECTS) and "Storia contemporanea” (5 ECTS). The 60 ECTS path includes, in addition to the three above mentioned compulsory modules, a module of "Mathematical language and Problem Solving" (10 ECTS) which is mandatory, and two modules of 10 ECTS each that can be chosen among "Cinema and Music", "Law and Economics" "Languages and Cultures of the World" and "Sciences". The compulsory nature chosen for some of the modules is in line with the educational objectives defined during the design phase; the skills, abilities and knowledge provided by the compulsory courses of both paths are the minimum requirements not only for the enrollment at the university, but also for the education of the citizen and to facilitate the inclusion of students in the university and in the social sphere of our country. The first 10 ECTS regarding the "Italian Language and Culture " module will be available for free, but registration will be required in order to view the rest of the contents of both paths. The student will receive a certificate stating the passing of the final online exam of each module which will allow him/her to take the face to face exam in Turin. The latter will consist of two distinct parts: the first one, computer-based and related to scientific and economic-juridical lessons, will be carried out with the same automatic evaluation tool that the students got to know during the online course; the second one, oral and related to humanistic subjects, will verify the linguistic skills acquired by the student at the end of the course.

\section{Results and discussion}

\subsection{Connection from secondary school to university}

One of the main results in the medium and long term that we expect from the Foundation Program is the strengthening of the link between secondary school and university, reinforced with other projects of the University of Turin at national level. This international dimension represents a possibility for any student interested in coming to Europe for study purposes to fill any gaps in the easiest way possible, and to allow them to access the Italian university gradually but consciously. Other goals that we hope to achieve, also through the spreading of the initiative via various channels such as embassies and international fairs, is to offer the possibility to get to know Italian culture or to deepen the knowledge at a pre-university level. 
Courses will be made available at the end of March 2019, so we will not be able to know how many students will attend them, and what nationality they are, for another year.

\subsection{Professional training of university teachers}

A result that has already been achieved is the training of the 18 university teachers who are in charge of the 8 modules. They were trained both in the use of educational methodologies that are specific in e-learning (communication strategies with videos, docimological training, design and implementation of on-line courses) and in the technical skills that concern content creation (ACE, AAS, video editing tools); thus, they acquired skills that can be useful beyond and after this project, as they can be employed in regular didactic too. These teachers are to be added to other university teachers who were already trained during other projects and who contribute to a deep innovation in university education. As for the preparation of materials, each teacher was supported by a grant holder who had been properly trained, because the teacher worked extra hours and beyond his/her regular tasks for this project.

\subsection{Examples of digital educational materials}

All the courses are currently under revision and completion. In the initial phases, a template for structuring the ECTS was recommended (introductory video, interactive material, formative evaluation, in-depth analysis and summative assessment), but during the implementation phase teachers were given complete freedom as of the elaboration of the various parts, according to the needs of the single subjects. The only specific request was constant interactivity, which had to be used both for the resources and for the LMS Moodle activities. The search for the most suitable solutions for the creation of interactive materials that allow the student to independently reach a specific educational objective has been particularly challenging in some cases, and has led to the discovery of new and interesting teaching tricks. Some examples of activities used are: tests with automatic evaluation consisting of adaptive questions with personalized feedback, interactive worksheets for the exploration of contextualized problematic situations created with an ACE that should develop problem solving skills (Barana, Fioravera, Marchisio, 2017c; Barana, Fioravera, Marchisio, 2017d), Moodle lessons. Examples of resources are: video lessons, interactive pdfs, and compilable Moodle pages. 


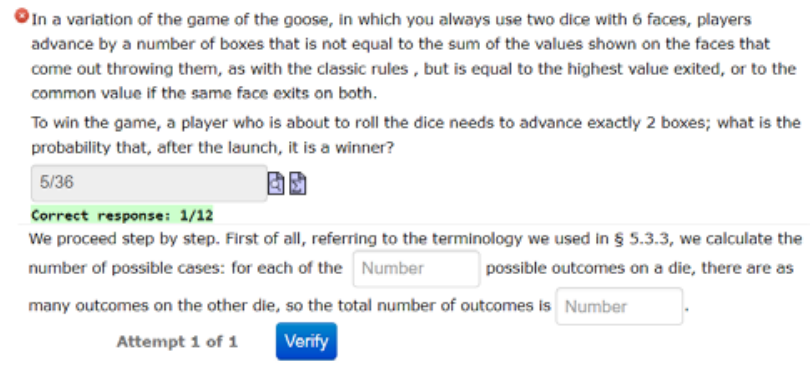

Figure 1. An example of adaptive question.

Figure 1 shows an adaptive question of a test for the "Mathematical Language and Problem Solving” course, concerning the solving of a probability problem. The question is built with an interactive feedback, (Barana et al., 2018b) this meaning that, if the student gives an incorrect answer to the first question, a guided step by step procedure is proposed until the whole problem is solved.

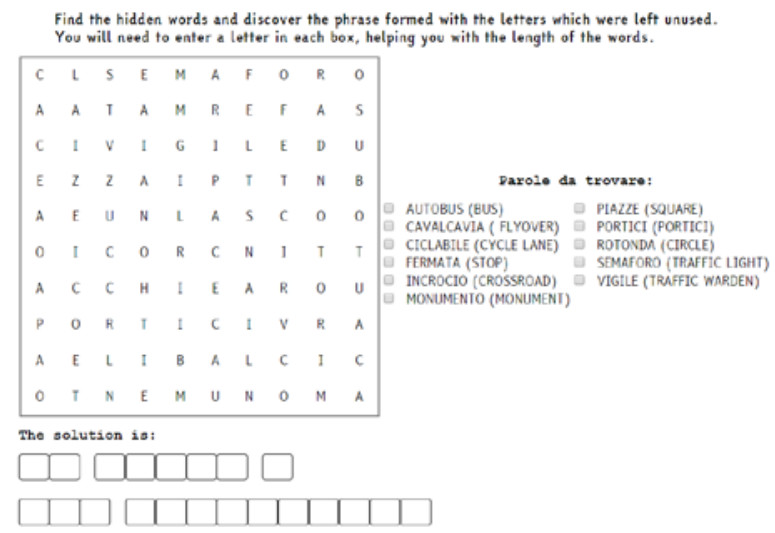

Figure 2. An example of Moodle's page.

Figure 2 shows an example of wordsearch built on a page which allows the students to highlight the words in the box, to check the words found and to enter the solution in the appropriate area. This resource is proposed in the "Playground" under the Italian Language and Culture section. As for the History course, we were given access to the archives of the Library of the Italian Risorgimento Museum, the "Vera Nocentini" Foundation and the "Gaetano Salvemini" Institute of Historical Studies, which authorized the publication of unpublished materials, thus giving a remarkable originality to teaching.

The Foundation Program will be available not only to foreign students who need to obtain the twelfth year of schooling, but to all those who want to study, by purchasing the entire 
course as a single 60 or 30 ECTS. Several requests have already been received from interested students from various foreign countries, such as Azerbaijan, Iran, Brazil and Egypt, driven mostly by the online feature and all the advantages that it entails. We can affirm that a bridge has been built to allow a real and modern internationalization of the university, which also means to approach university in this case the Italian one, in the best way possible.

\section{References}

Barana, A., Bogino, A., Fioravera, M., Floris, F., \& Marchisio, M. (2018a). Realignment Course in Mathematics: design of an online valuable experience for students. Proceedings of the 4th International Conference on HEAd'18, 1465-1473. Univ. Politècnica València.

Barana, A., Bogino, A., Fioravera, M., Floris, F., Marchisio, Operti, L., M., Rabellino, S., (2017a). Self-Paced Approach in Synergistic Model for Supporting and Testing Students. In Proceedings of 2017 IEEE 41st COMPSAC, 407-412. Turin: IEEE.

Barana, A., Bogino, A., Fioravera, M., Marchisio, M., \& Rabellino, S. (2016a). Digital Support for University Guidance and Improvement of Study Results. Procedia - Social and Behavioral Sciences, 228, 547-552. https://doi.org/10.1016/j.sbspro.2016.07.084

Barana, A., Brancaccio, A., Esposito, M., Fioravera, M., Marchisio, M., Pardini, C., \& Rabellino, S. (2017b). Problem solving competence developed through a virtual learning environment in a european context. In Proceedings of International Scientific Conference eLearning and Software for Education, 455-463, Bucharest: ADLRO.

Barana, A., Conte, A., Fioravera, M., Marchisio, M., \& Rabellino, S. (2018b). A Model of Formative Automatic Assessment and Interactive Feedback for STEM. Proceedings of 2018 IEEE 42nd COMPSAC, 1016-1025. Tokyo, Japan: IEEE.

Barana, A., \& Marchisio, M. (2016b). Ten Good Reasons to Adopt an Automated Formative Assessment Model for Learning and Teaching Mathematics and Scientific Disciplines. Procedia - Social and Behavioral Sciences, 228, 608-613.

Barana, A., Marchisio, M., \& Rabellino, S. (2015). Automated Assessment in Mathematics. Proceedings of 2015 IEEE 39th COMPSAC, 670-671.

Barana, A., Fioravera, M., \& Marchisio, M. (2017c). Developing problem solving competences through the resolution of contextualized problems with an Advanced Computing Environment, 1015-1023. Proceedings of the 3rd International Conference on Head'17. Univ. Politècnica València. https://doi.org/10.4995/HEAD17.2017.5505

Barana, A., Fioravera, M., \& Marchisio, M. (2017d). Teacher training: a model for introducing innovative digital methodologies for learning Mathematics. Proceedings of the 3rd International Conference on Head'17, 608-616.

Barana, A., Fioravera, M., Marchisio, M., \& Rabellino, S. (2017e). Adaptive Teaching Supported by ICTs to Reduce the School Failure in the Project "Scuola Dei Compiti". Proceedings of 2017 IEEE 41st COMPSAC, 432-437.

Brancaccio, A., Esposito, M., Marchisio, M., \& Pardini, C. (2016). L’efficacia dell'apprendimento in rete degli immigrati digitali. L'esperienza SMART per le discipline scientifiche. MONDO DIGITALE, 15, 803-821. 
Brancaccio, A., Marchisio, M., Palumbo, C., Pardini, C., Patrucco, A., \& Zich, R. (2015). Problem Posing and Solving: Strategic Italian Key Action to Enhance Teaching and Learning Mathematics and Informatics in the High School. Proceedings of 2015 IEEE 39th COMPSAC, 845-850. Taichung, Taiwan: IEEE.

Bruschi, B., Cantino, V., Cavallo Perin, R., Culasso, F., Giors, B., Marchisio, M., Marello, C., Milani, M., Operti, L., Parola, A., Rabellino, S., \& Scomparin, L. (2018). Start@unito: a Supporting Model for High School Students Enrolling to University, 307-312. Presented at IADIS Int. Conf. Cognition and Exploratory Learning in Digital Age 2018.

Burbules, N. C., \& Torres, C. A. (2000). Globalization and education: critical perspectives. New York: Routledge.

Czerkawski, B. C., \& Lyman, E. W. (2016). An Instructional Design Framework for Fostering Student Engagement in Online Learning Environments. TechTrends, 60 (6), 532-539. https://doi.org/10.1007/s11528-016-0110-z

Dixson, M. D. (2010). Creating effective student engagement in online courses: What do students find engaging? J. of the Scholarship of Teaching and Learning, 10 (2), 1-13.

Isaxanli, H. (2018). Education Facing Globalization in Post-communist Country: Azerbaijan. Proceedings of the 4th International Conference on HEAd'18.Univ. Politècnica València.

Marchisio, M., Operti, L., Rabellino, S., \& Sacchet, M. (2018). Start@unito: Open Online Courses for Improving Access and for Enhancing Success in Higher Education. In CSEDU, 639-646.

MIUR. Procedure per l'ingresso, il soggiorno e l'immatricolazione degli studenti richiedenti visto ai corsi della formazione superiore in Italia, anno accademico 2018-2019 (2018). 